\title{
Acute Dystonia after Using Single Dose Duloxetine: Case Report
}

\author{
Görkem Karakaș Uğurlu' ${ }^{\bowtie}$, Sinay Önen², Deniz Bayındırlı and Ali Çayköylü ${ }^{3}$ \\ 1'Department of Psychiatry, Ankara Atatürk Training and Research Hospital, Ankara, Turkey \\ ${ }^{2}$ Department of Psychiatry, Bartın State Hospital, Bartın, Turkey \\ ${ }^{3}$ Department of Psychiatry Ankara, Yıldırım Beyazıt University Ankara Atatürk Training and Research Hospital, Turkey
}

Duloxetine is a balanced and potent serotonin and noradrenaline reuptake inhibitor which is known to be effective in depression and anxiety disorders. The common adverse effects include dry mouth, nausea, insomnia, somnolence, dizziness and constipation. Reported adverse effects of the extra pyramidal symptoms (EPS) are rare. In this case, a patient who suffered from acute dystonia, after only one dose of $30 \mathrm{mg}$ duloxetine is presented.

Psychiatry Investig 2013;10:95-97

Key Words Duloxetine, Dystonia.

\section{INTRODUCTION}

Duloxetine is a balanced and potent serotonin and noradrenaline reuptake inhibitor which is known to be effective in depression and anxiety disorders. ${ }^{1-3}$ Common adverse effects include dry mouth, nausea, insomnia, somnolence, dizziness and constipation. Reported adverse effects of EPS are rare., ${ }^{4,5}$

Acute dystonia is characterized by prolonged muscle contraction, frequently presenting with marked head and neck muscle spasm with occasional jaw clenching and temporomandibular joint syndrome. Although, it is believed that the dystonia results from the abnormality of the basal ganglia, the pathophysiology of acute dystonia is not clear yet. However, a few cases of duloxetine induced dystonia are reported in the literature. ${ }^{5-7}$ In this case, a patient who suffered from a neck spasm and pain which woke her up from sleep, dystonic distortion of the neck, tremor in her hands and lips after only one dose of $30 \mathrm{mg}$ duloxetine is presented.

\section{CASE}

Miss. M. is a 19-year-old, female patient. She was referred to

Received: March 21, 2012 Revised: June 4, 2012

Accepted: October 5, 2012 Available online: February 8, 2013

$\triangle$ Correspondence: Görkem Karakaş Uğurlu, MD

Department of Psychiatry, Ankara Atatürk Training and Research Hospital, Üniversiteler street, Bilkent boulevard, 06800 Ankara, Turkey

Tel: +9003122912525, Fax: +9003122912705, E-mail: dr_gorkem@yahoo.com

(a) This is an Open Access article distributed under the terms of the Creative Commons Attribution Non-Commercial License (http://creativecommons.org/licenses/bync/3.0) which permits unrestricted non-commercial use, distribution, and reproduction in any medium, provided the original work is properly cited. the emergency service with a sudden onset of severe neck spasm and pain, distortion of head, mild tremor in her hands and lips, mild pouting, movement of forehead and jaw cleching. The patient was aware of this situation and was in severe distress. She said that her complaints have started after 10 hours of taking only one dose of duloxetine $30 \mathrm{mg}$. Duloxetine $30 \mathrm{mg}$ /day had been prescribed by another psychiatrist for her anxiety symptoms. She did not take any medication except duloxetine $30 \mathrm{mg}$ /day. She did not have any history of head trauma, seizure, medical disease, substance abuse or family history of neuropsychiatric disorders.

The clinical presentation was interpreted as dystonia and Abnormal Involuntary Movement Scale (AIMS) was performed. The AIMS score was 22. Within 30 minutes after parenteral $5 \mathrm{mg}$ biperiden administration, all signs and symptoms improved and dystonia were not observed throughout the 6-hour emergency service monitoring. During her treatment, a series of laboratory tests was performed to rule out secondary dystonia. These tests included complete blood count, erythrocyte sedimentation rate, liver, renal and thyroid function tests, blood glucose level, serum copper, zinc, iron, magnesium, ceruloplasmin level, total iron binding capacity, serum ferritin, vitamin B12, folic acid level, cranial magnetic resonance imaging and electroencephalogram. These examinations showed no specific findings. Thus, this manifestation was evaluated as duloxetine induced dystonia. Duloxetine was stopped. She regularly attended psychotherapy for anxiety disorder, not otherwise specified according to diagnostic criteria of DSM-IV. She was followed up six months without medication. Anxiety symptoms were not observed after psh- 
chotherapy and also dystonia did not appear again during this period.

\section{DISCUSSION}

Medication induced acute dystonia can be a side effect of treatment with antipsychotics, antidepressants, antiemetics and other drugs. Its risk factors include male gender, young age, high dose and high potency antipsychotics and parenteral administration of antipsychotics. ${ }^{8-11}$

The pathophysiology of dystonia is not clear yet. Since acute dystonia is associated with antipsychotic medication, the hypothesis of dopaminergic system disturbance or impairment in dopamine-acetylcholine balance are commonly believed to be the underlying reason. Besides antipsychotic drugs, antidepressant agents may also cause either an increase or a decrease (through serotonin receptors that inhibit dopaminergic pathways) in dopamine levels resulting in movement disorders. ${ }^{12}$ Additionally, the serotonergic system is considered to have complicated interactions with various receptor subtypes such as gamma amino butyric acid, and cholinergic system. Tonic inhibitor effect on dopaminergic functions via serotonergic neurons in brain stem and impairment in acethylcholine-dopamine balance are thought to be responsible for selective serotonin reuptake inhibitor (SSRI) associated acute dystonia. ${ }^{13}$ There have been several case reports of acute dystonia in patients who treated with antidepressant agents such as fluvoxamine, sertraline, fluoxetine, citalopram, mirtazapine, paroxetine, venlafaxine and bupropion., ${ }^{3,14-16}$ In addition, duloxetine-induced tardive dystonia and tardive dyskinesia case was reported in literature. ${ }^{17}$ In a review, U.S. Food and Drug Administration Adverse Event Reporting System was researched and it was found that 89 patients with antidepressant induced EPS had been reported between 20052008. 59 of the reported cases were due to duloxetine, and 6 of them were acute dystonia. ${ }^{18}$ This manifestation points out that, duloxetine is a more remarkable antidepressant than the others, in terms of acute dystonia. The pathophysiologic basis of dystonia remains obscure. Interconnection between the serotonergic and dopaminergic systems seems to play a major role. As a result of duloxetine strongly inhibits neuronal reuptake of serotonin and norepinephrine as shown in preclinical studies, increased serotonin transmission may inhibit dopaminergic neurotransmission. ${ }^{19}$ Additionally, dystonia results from impairment of a normal dopaminergic-noradrenergic balance, in which noradrenergic tone preponderates. A relative norepinephrine hyperactivity may be caused by dopaminergic blockade (as occurs in drug-induced dystonia) or from enhanced release of norepinephrine (in idiopathic torsion dystonia). ${ }^{20}$ Because of the inhibition of norepinephrine reup- take and increased norepinephrine, dystonia can be occur more with duloxetine than SSRI's. However, the role of single dose of duloxetine on the neurotransmitter system and its role in the appearance of dystonia needs to be supported in latter studies.

Although there have been few reports of antidepressant induced acute dystonia; ${ }^{3,14-16}$ this painful, terrifying and sometimes life-threatening side effect, which needs early detection and management, these side effect can cause disturbance of the relationship between the physician and the patient. ${ }^{10} \mathrm{Cli}$ nicians should be aware of the potency to cause EPS for all classes of antidepressant agents.

\section{REFERENCES}

1. Lai CH. Duloxetine related binge eating behaviors: a case report. Prog Neuropsychopharmacol Biol Psychiatry 2009;33:1581-1582.

2. Park YM, Lee BH, Lee HJ, Kang SG. Cholestatic jaundice induced by duloxetine in a patient with major depressive disorder. Psychiatry Investig 2010;7:228-230.

3. Arnone D, Hansen L, Kerr JS. Acute dystonic reaction in an elderly patient with mood disorder after titration of paroxetine: possible mechanisms and implications for clinical care. J Psychopharmacol 2002;16: 395-397.

4. Westanmo AD, Gayken J, Haight R. Duloxetine: a balanced and selective norepinephrine- and serotonin-reuptake inhibitor. Am J Health Syst Pharm 2005;62:2481-2490.

5. Deuschle M, Mase E, Zink M. Dyskinesia during treatment with duloxetine. Pharmacopsychiatry 2006;39:237-238.

6. Detweiler MB, Harpold GJ. Bupropion-induced acute dystonia. Ann Pharmacother 2002;36:251-254.

7. Sutton AL. Mental Health Disorders Sourcebook: Basic Consumer Health Information about the Causes and Symptoms of Mental Health Problems, Including Depression, Bipolar Disorder, Anxiety Disorders, Post-Traumatic Stress Disorder, Obsessive-Compulsive Disorder, Eating Disorders, Addictions, and Personality and Schizophrenic Disorders. United States: Omnigraphics Inc.; 2009.

8. Wang HY, Chou WJ, Huang TY, Hung CF. Acute dystonia resulting from abrupt bupropion discontinuation. Prog Neuropsychopharmacol Biol Psychiatry 2007;31:766-768.

9. Keepers GA, Casey DE. Prediction of neuroleptic-induced dystonia. J Clin Psychopharmacol 1987;7:342-345.

10. van Harten PN, Hoek HW, Kahn RS. Acute dystonia induced by drug treatment. BMJ 1999;319:623-626.

11. Kısa C, Aydemir Ç, Göka E. Temporomandibular joint dislocation due to orofacial dystonia during antipsychotic use: a case. J Clin Psychiatry 2007;10:102-105.

12. Ng J, Sansone RA, McDonald S. Akathisia and abnormal movements of the upper extremities with venlafaxine and methimazole. Gen Hosp Psychiatry 2009;31:388-390.

13. Wilkinson G. Seminars in general adult psychiatry. Trowbridge, Wiltshire, UK: Royal College of Psychiatrists; 2007.

14. Lee Y, Yeh WC, Chong MY, Lin PY, Chang YY. Venlafaxine and tardive blepharospasm: a case report. Prog Neuropsychopharmacol Biol Psychiatry 2007;31:1139-1140.

15. George MS, Trimble MR. Dystonic reaction associated with fluvoxamine. J Clin Psychopharmacol 1993;13:220-221.

16. Dave M. Fluoxetine-associated dystonia. Am J Psychiatry 1994;151:149.

17. Chen PY, Lin PY, Tien SC, Chang YY, Lee Y. Duloxetine-related tardive dystonia and tardive dyskinesia: a case report. Gen Hosp Psychiatry 2010;32:646. 
18. Madhusoodanan S, Alexeenko L, Sanders R, Brenner R. Extrapyramidal symptoms associated with antidepressants--a review of the literature and an analysis of spontaneous reports. Ann Clin Psychiatry 2010; 22:148-156.
19. Leo RJ. Movement disorders associated with the serotonin selective reuptake inhibitors. J Clin Psychiatry 1996;57:449-454.

20. Korczyn AD. The pathophysiology of dystonia. J Neural Transm 1978; 42:245-250. 